\title{
Synthesis, characterization and photoluminescence properties of tetra(aminophenyl) porphyrin covalently linked to multi-walled carbon nanotubes
}

\author{
G PRABHAVATHI ${ }^{\mathrm{a}}$, M ARJUN ${ }^{\mathrm{b}}$ and R YAMUNA ${ }^{\mathrm{b}, *}$ \\ ${ }^{a}$ Research and Development Centre, Bharathiar University, Coimbatore, Tamil Nadu 641 046, India \\ ${ }^{\mathrm{b}}$ Department of Chemical Engineering and Material Science, Department of Sciences, Center of Excellence \\ in Advanced Materials and Green Technologies (CoE-AMGT), Amrita School of Engineering, Amrita Vishwa \\ Vidyapeetham, Amrita University, Coimbatore, Tamil Nadu 641 112, India \\ E-mail: ryamuna1@gmail.com
}

MS received 9 February 2017; revised 4 May 2017; accepted 5 May 2017

\begin{abstract}
Synthesis of a new nano hybrid of 5,10,15,20-mesotetra(4-aminophenyl) porphyrin (TAP) functionalized with multi-walled carbon nanotubes (MWCNTs) through an amide linkage is reported for the first time. This MWCNT-TAP hybrid was characterized by Raman, Fourier transform infrared (FT-IR), Transmission electron microscopy (TEM), Thermogravimetric analysis (TGA), absorption and emission spectroscopy. TGA analysis reveals that there is a $\sim 60 \%$ weight loss when heated from $150-750^{\circ} \mathrm{C}$, which is attributed to the amount of TAP molecules that were attached to MWCNTs. Electronic properties of MWCNTs were improved in the hybrid compared to raw MWCNTs as evidenced by Raman spectra. The absorption and emission spectra of TAP and nano-hybrid indicate strong positive solvatochromism with increasing solvent polarity. Fluorescence quenching of TAP in different solvents were observed in the emission spectra in the MWCNT-TAP hybrid, indicating that covalent functionalization facilitated effective energy or electron transfer from porphyrin moiety to the MWCNT.
\end{abstract}

Keywords. MWCNT-TAP; photoluminescence; solvatochromism; fluorescence quenching.

\section{Introduction}

Carbon nanotubes (CNTs) have attracted vast attention in recent years owing to their exceptional electrical, chemical and mechanical properties, which make them excellent candidates for various applications. ${ }^{1-5}$ Multiwalled carbon nanotubes (MWCNTs) are usually composed of a large number of concentric carbon nanotubes. For this reason, MWCNTs are considered as one of the best candidates among the carbon nanomaterials as a component of electron donor-acceptor (D-A) ensembles. ${ }^{6,7}$ Therefore, significant effort has been devoted to the chemical functionalization of MWCNTs, mainly to enhance their solubility and to improve their compatibility in MWCNT-based nanohybrid systems. ${ }^{8-10}$ Linking of electron donor such as porphyrin chromophore to MWCNT as acceptor (D-A) through a covalent bond is expected to facilitate the development of hybrid materials with improved solubility and photoelectronic properties through a synergistic

\footnotetext{
*For correspondence
}

effects. ${ }^{11-13}$ Porphyrins are thermally stable, light harvesting molecules that show excellent nonlinear optical properties owing to their extensive $\pi$-electron delocalization. Incorporation of photoexcited electron donors, such as porphyrins or metal phthalocyanine macrocyles, with carbon nanomaterials would constitute an ideal candidate for photovoltaic and fuel cell applications. ${ }^{10,12,14}$ In the last few years, numerous studies have been reported in the literature on non-covalent porphyrin-nanocomposites formed due to $\pi-\pi$ and van der Waals forces for various molecular photoelectronic applications. ${ }^{15-19}$ Nevertheless, studies involving covalent incorporation of porphyrin chromophores with carbon nanomaterials are limited. ${ }^{20-25}$ Moreover, covalently functionalized porphyrin with carbon nanomaterials have two disadvantages; first one is low degree of porphyrin functionalization on the surface of CNTs, and another is that the structure of CNTs is badly damaged during the carboxylation of MWCNTs surface by strong oxidative acid treatment. On the other hand, the reports on the functionalization of CNTs with porphyrin molecules to surmount these two weaknesses all together are scarce. ${ }^{21}$ Most of the porphyrin-linked 
carbon nanomaterials reported to date contain only unsubstituted mesotetraphenyl porphyrin (TPP). ${ }^{21,26-28}$ In this paper, we report the synthesis of a new nanohybrid of MWCNT that has been covalently functionalized with 5,10,15,20-mesotetra(4-aminophenyl) porphyrin (TAP) through an amide linkage. TAP has more potential compared to TPP as it has free amino groups (electron donating) that can enhance the optical properties of the nanohybrid. Synthesized MWCNT-TAP has been characterized by thermogravimetric analysis (TGA), Transmission electron microscopy (TEM), Raman, Fourier transform infrared (FT-IR), absorption and emission spectroscopy.

\section{Experimental}

\subsection{Materials and characterization}

Pyrrole, triethylamine, p-nitrobenzaldehyde, and tin(II) chloride dihydrate were purchased from Sigma Aldrich. Pyrrole was purified by vacuum distillation. Other reagents such as chloroform $\left(\mathrm{CHCl}_{3}\right)$, dry tetrahydrofuran (THF, Finar) and $\mathrm{N}, \mathrm{N}$ - dimethyl formamide (DMF) were purified by using standard procedures. MWCNTs were purchased from Yunnan Great (Group) Co., Ltd. (China). Carboxyl functionalized MWCNTs (MWCNT-COOH) and TAP were prepared by using the literature procedure. ${ }^{20,29}$ Absorption spectrum was measured with SHIMADZU, UV-1800 and SHIMADZU UV2600 spectrophotometers. Fourier transform infrared spectrum (FTIR) was taken using the $\mathrm{KBr}$ pellet method on a Thermo Nicolet, iS10 FTIR spectrophotometer. Raman spectrum was measured using micro Raman spectroscopy (Princeton instrument Acton sp2500). Fluorescence spectrum was recorded using a SHIMADZU RF-6000 computer aided spectrofluorophotometer. Quantum yield of the substances were measured in DMF at $20^{\circ} \mathrm{C}$, using $1 \mathrm{~cm} \mathrm{x} 1 \mathrm{~cm}$ quartz optical cells under aerobic conditions. For measurements of quantum yields $\left(\Phi_{\mathrm{F}}\right)$, TPP in DMF was used as reference $\left(\Phi_{\mathrm{F}}=0.11\right) .{ }^{30}$ Thermogravimetric analysis (TGA) was performed using SDT Q600 simultaneous DSC-TGA with a heating rate of $5^{\circ} \mathrm{C} / \mathrm{min}$ under $\mathrm{N}_{2}$ purge $(100 \mathrm{~mL} / \mathrm{min})$. JEOL JEM 2100 high resolution transmission electron microscopy (HR-TEM) instrument was used to record the TEM images of nanohybrid.

\subsection{Synthesis of TAP-NHCO-MWCNT nanohybrid}

MWCNT-COOH (100 mg) was refluxed at $65^{\circ} \mathrm{C}$ for $24 \mathrm{~h}$ with $\mathrm{SOCl}_{2}(75 \mathrm{~mL})$ under argon atmosphere in the presence of DMF $(1.8 \mathrm{~mL})$ to form acyl chloride functionalized MWCNT (MWCNT-COCl). Excess $\mathrm{SOCl}_{2}$ was removed by vacuum distillation and the remaining residue was washed with dry THF. MWCNT-COCl (100 mg) and TAP (100 $\mathrm{mg}$ ) were dissolved in DMF (40 mL). Further this mixture was refluxed at $130^{\circ} \mathrm{C}$ for $72 \mathrm{~h}$ in presence of $1 \mathrm{~mL}$ triethylamine $\left(\mathrm{Et}_{3} \mathrm{~N}\right)$ under argon atmosphere. The resultant solution was cooled to room temperature. Excess DMF was removed by using rotary evaporator. $100 \mathrm{~mL}$ of ether was added into the reaction mixture in order to precipitate the product. The residue was separated through ultracentrifugation. Finally, the precipitate was purified through three washing cycles each consisting of ultra centrifugation in THF, followed by filtration through nylon filter membrane in order to remove excess TAP and other impurities from the residue. The resultant product was washed with $\mathrm{CHCl}_{3}$ by following the above procedure and $\mathrm{Et}_{3} \mathrm{~N}$. $\mathrm{HCl}$ was removed from the nanohybrid by washing it with water and it was dried under vacuum.

\section{Results and Discussion}

\subsection{Synthesis of materials}

Carboxyl-functionalized MWCNT (MWCNT-COOH) was synthesized by sonicating MWCNT with concentrated solution of $\mathrm{HNO}_{3} / \mathrm{H}_{2} \mathrm{SO}_{4}(1: 3 \mathrm{ratio}$, v/v) at $60^{\circ} \mathrm{C}$ for $4 \mathrm{~h}$ according to a slightly modified literature procedure. ${ }^{29}$ Further, this was stirred for $9 \mathrm{~h}$ at room temperature. The resultant MWCNT dispersion was filtered through a nylon filter membrane. The final residue was continuosly washed using ultrapure water (18 M $\Omega$, Milli-Q, Millipore) until the $\mathrm{pH}$ of the filtrate was 7 and then dried overnight in a oven at $80^{\circ} \mathrm{C}$. The synthesis of TAP was carried out by reducing 5,10,15,20-mesotetra(4-nitrophenyl) porphyrin (TNP) using the literature procedure and further purified by soxhlet extraction. ${ }^{20}$ Precursor TNP was synthesized from 4-nitrobenzaldehyde and pyrrole by using Alder's method. ${ }^{31}$ TAP (100 mg) was covalently linked to MWCNT through an amide bond by refluxing it with MWCNT-COCl $(100 \mathrm{mg})$ at $130^{\circ} \mathrm{C}$ for $72 \mathrm{~h}$ in DMF solvent under argon in the presence of triethylamine $\left(\mathrm{Et}_{3} \mathrm{~N}\right)$ as shown in the Scheme 1. Though there are four free $\mathrm{NH}_{2}$ groups in TAP, not all could form amide bond with MWCNT, may be due to the tubular nature of the MWCNT. This was confirmed by us by doing the independant experiments with less quantity of TAP (30 $\mathrm{mg}$ ), we ended up with the poor solubility of the resultant MWCNT-TAP nanohybrid. This may be due to the low degree of functionalization of TAP molecules with MWCNT.

\subsection{Spectroscopic and thermal studies}

Figure 1 shows the chemical structure of nanohybrid. MWCNT-TAP hybrid was characterized by FT-IR spectrum in the range of $500-4000 \mathrm{~cm}^{-1}$ as shown in 


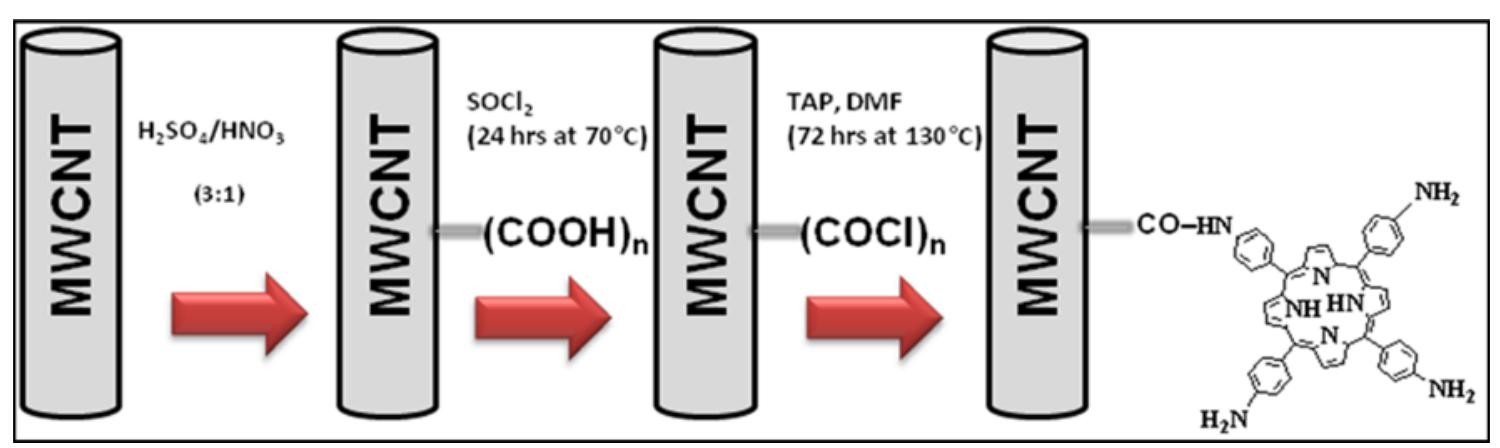

Scheme 1. Reaction scheme for the covalent functionalization of porphyrin with MWCNTs.

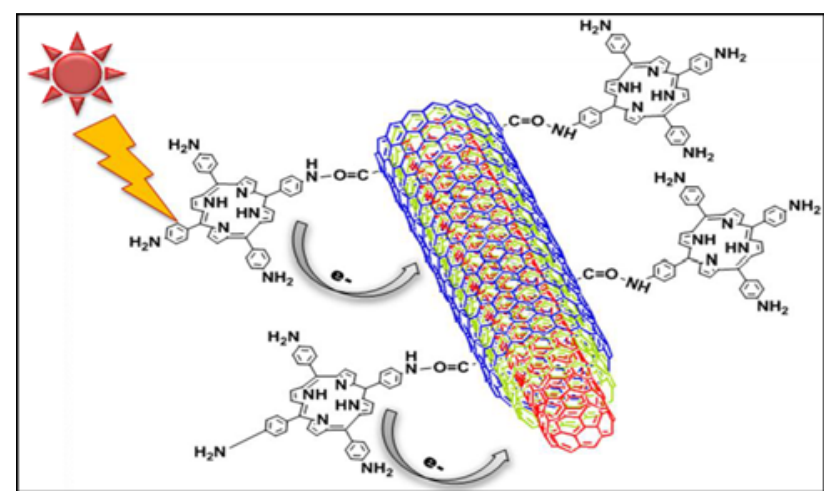

Figure 1. Chemical structure of MWCNT-TAP.

Figure 2. In the FT-IR spectrum, MWCNT-COOH has characteristic absorption peak at $3414 \mathrm{~cm}^{-1}$ which corresponds to the $\mathrm{O}-\mathrm{H}$ vibration frequency. Carbonyl $(\mathrm{C}=\mathrm{O})$ stretching vibration of the $\mathrm{COOH}$ group appears at $1709 \mathrm{~cm}^{-1}$. Three new striking peaks appeared in the hybrid, after covalent functionalization with porphyrin, at $1638 \mathrm{~cm}^{-1}, 1211 \mathrm{~cm}^{-1}$ and $1597 \mathrm{~cm}^{-1}$ which correspond to the formation of $\mathrm{C}=\mathrm{O}, \mathrm{C}-\mathrm{N}$ of amide linkage $(-\mathrm{CO}-\mathrm{NH})$, and the stretching vibration frequency of porphyrin $\mathrm{C}=\mathrm{C}$, respectively. ${ }^{27,32}$ TAP shows two N-H stretching frequencies at $3336 \mathrm{~cm}^{-1}$ and $3428 \mathrm{~cm}^{-1}$ due to $\mathrm{N}-\mathrm{H}$ stretching of amino group $\left(-\mathrm{NH}_{2}\right)$ and porphyrin NH group whereas MWCNT-TAP hybrid shows a single broad band at $3438 \mathrm{~cm}^{-1}$ owing to the merging of all three $\mathrm{N}-\mathrm{H}$ stretchings $\left(\left(-\mathrm{CO}-\mathrm{NH},-\mathrm{NH}_{2}\right.\right.$ and porphyrin NH groups). These changes in the FT-IR spectra clearly indicate the formation of covalently connected MWCNT-TAP nano hybrid.

TGA curves of MWCNT-TAP, MWCNT-COOH, pure MWCNT and TAP are shown in the Figure 3. The TGA curve of MWCNT-COOH indicates that it is a multi step processes as reported in the literature. ${ }^{33}$ The weight loss detected in the range of $50-180^{\circ} \mathrm{C}$ is attributed to the elimination of physisorbed water on the MWCNT-COOH surface (weight loss of $8 \%$ ). Further thermal decomposition is observed between $220-520^{\circ} \mathrm{C}$ (weight loss of 19\%). Such a weight loss may be attributed to the decarboxylation, decomposition of $\mathrm{OH}$ that are bound on the surface of MWCNT-COOH, degradation of disordered or amorphous carbon and other metal impurities. ${ }^{34}$ The TGA curve of MWCNTTAP shows that $\sim 60 \%$ weight loss when heated from $150-750^{\circ} \mathrm{C},{ }^{35}$ whereas the loss is much less $<20 \%$ for TAP and MWCNT, individually.

Figure 4 shows the Raman spectra of MWCNT, MWCNT-COOH and MWCNT-TAP. All these materials display characteristic $G$ and $D$ bands, with an $\mathrm{I}_{\mathrm{D}} / \mathrm{I}_{\mathrm{G}}$ ratio of $0.93,1.06$ and 0.80 , respectively. ${ }^{21}$ The $\mathrm{D}$ band reveals the presence of some disorder in the carbon nano structure. This band is frequently referred to as the defect

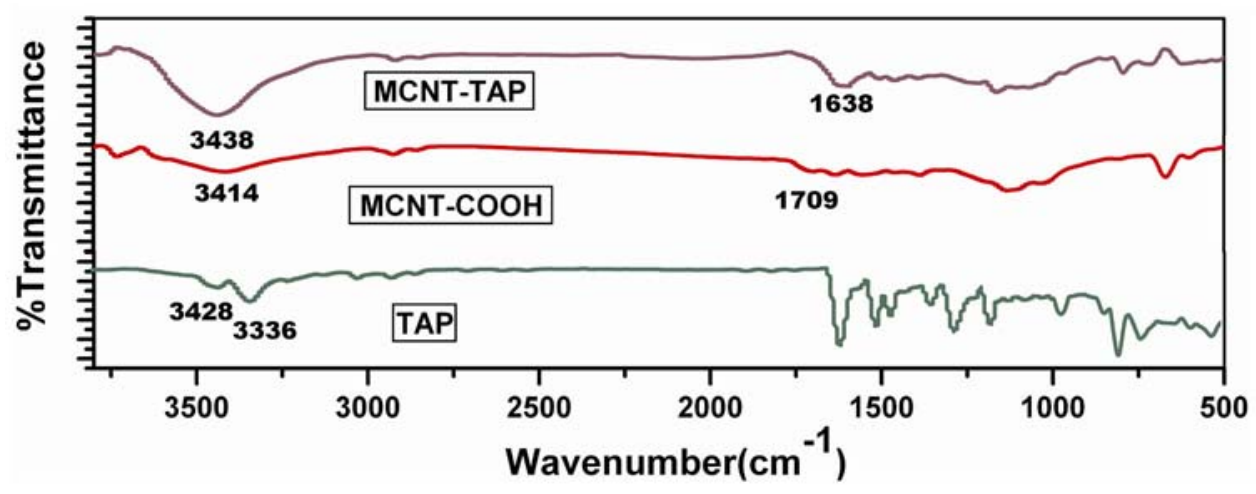

Figure 2. FT-IR spectra of TAP, MWCNTCOOH and MWCNT-TAP. 


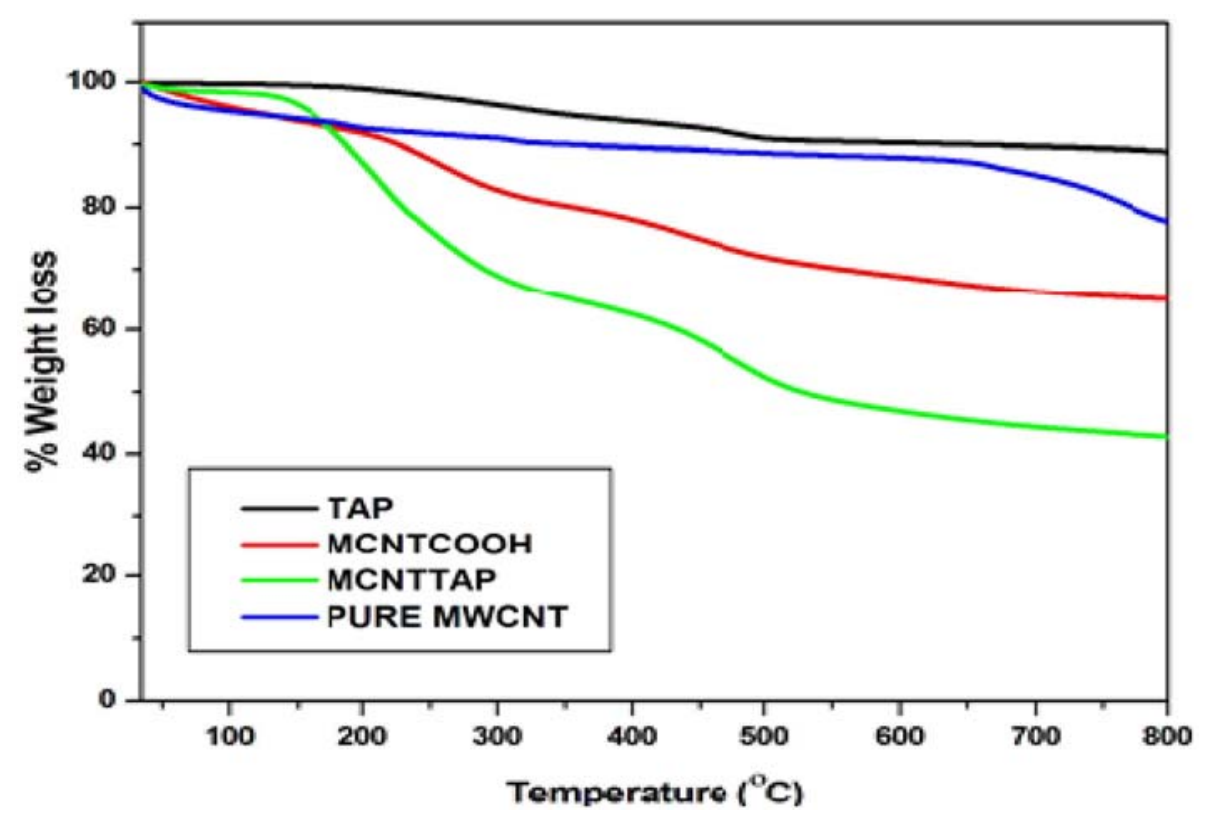

Figure 3. TGA curves of TAP, PURE MWCNT, MWCNT-COOH and MWCNT-TAP.

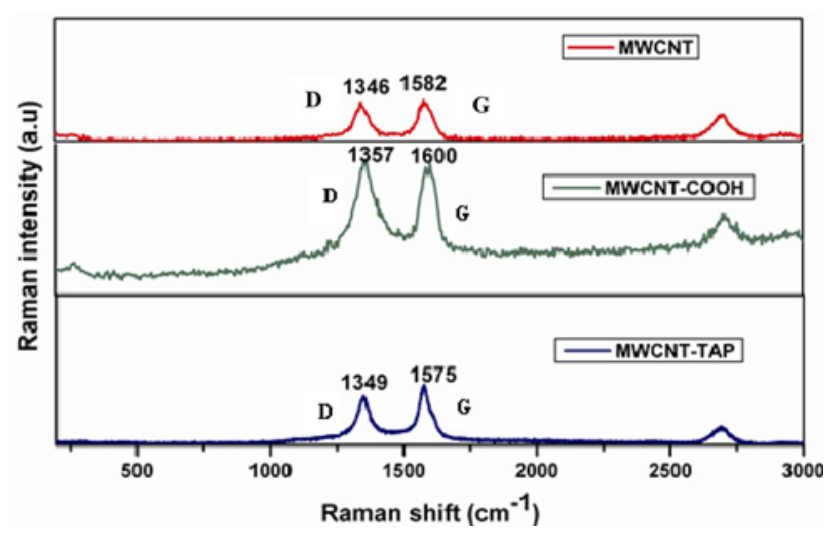

Figure 4. Raman spectra of MWCNT, MWCNT-COOH and MWCNT-TAP.

band. Its intensity relative to that of $\mathrm{G}$ band is often used to determine the quality of nanotubes. MWCNT$\mathrm{COOH}$ exhibited enhancement of the D peak compared to the MWCNT. Nevertheless, after functionalization with TAP, the intensity of the D band was significantly reduced compared with pure MWCNT. This indicates that the electronic properties of TAP-functionalized MWCNT were improved in the nanohybrid. Raman spectra of the MWCNT and its hybrid exhibit a band at $2700 \mathrm{~cm}^{-1}$ ( $\mathrm{G}^{\prime}$ band) which is characteristic band of multi-walled carbon nanotube. ${ }^{36} \mathrm{G}$ peak position of the MWCNT-TAP nanohybrid $\left(1575 \mathrm{~cm}^{-1}\right)$ exhibits a shift to lower frequency $\left(\approx 7 \mathrm{~cm}^{-1}\right)$ compared with MWCNT $\left(1582 \mathrm{~cm}^{-1}\right)$, whereas MWCNT-COOH $\left(1600 \mathrm{~cm}^{-1}\right)$ exhibits a higher frequency shift $\left(\approx 8 \mathrm{~cm}^{-1}\right)$ compared with MWCNT. In general, hybrid carbon nanomaterials exhibit this type of low frequency shift (softening) when functionalized with an electron donor molecule. It exhibits high frequency shift (stiffening) when hybridized with electron-acceptor molecule. ${ }^{37}$ Lower frequency shift in MWCNT-TAP hybrid confirms the charge transfer between TAP and MWCNT, where TAP acts as an electron donor and MWCNT acts as an electron acceptor. Higher frequency shift in MWCNT-COOH compared with MWCNT is possibly owing to the presence of electron withdrawing carboxyl group. This shift reveals the functionalization of MWCNTs with TAP.

Figure 5 shows UV-Vis absorption spectra for TAP and MWCNT-TAP nanohybrid in the range of 300 to $800 \mathrm{~nm}$ in DMF solvent. TAP reveals a strong Soret band at $436 \mathrm{~nm}$ and weak Q-bands between 500-700 $\mathrm{nm}$. MWCNT-TAP shows a blue shift in the Soret band (429 $\mathrm{nm}$ ) as compared to TAP. This result supports the covalent functionalization of TAP with MWCNT. Noncovalently attached porphyrins on MWCNTs do not cause shift or broadening of the Soret band. ${ }^{27}$ We observed that improved solubility in the case of MWCNT-TAP as compared to GO-TAP. The solutions of TAP, MWCNT-COOH, and MWCNT-TAP in DMF solvent are shown in Figure 6 (under visible light and long UV light $365 \mathrm{~nm}$ ). It reveals that dispersion of MWCNT-COOH in DMF solvent whereas MWCNTTAP was completely soluble in DMF solvent. Solution phase UV-Vis-NIR spectra were recorded to demonstrate a linear relationship between the absorbance and concentration of MWCNT and MWCNT-TAP. We have 


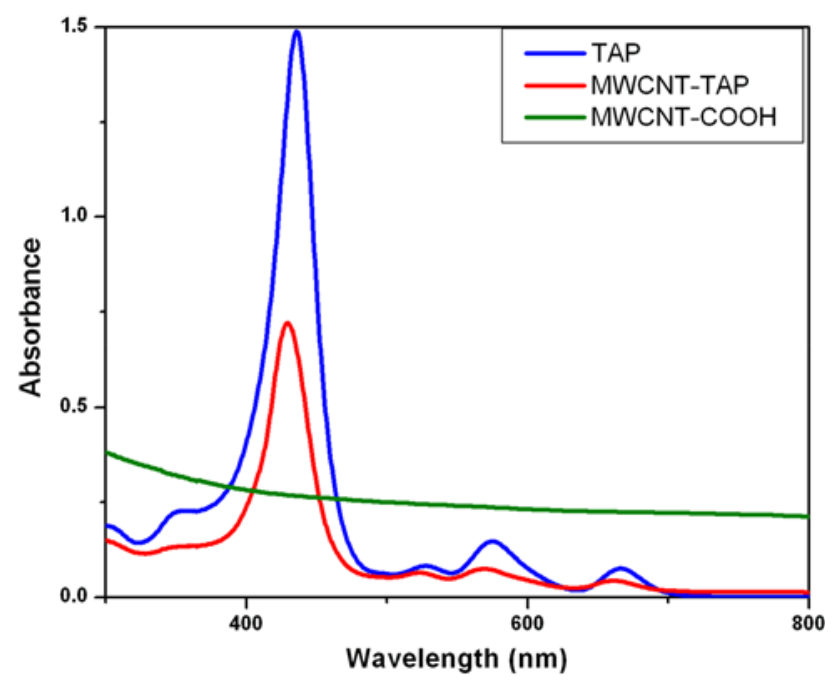

Figure 5. UV-Vis absorption spectra of TAP $(9.78 \mathrm{x}$ $\left.10^{-6} \mathrm{M}\right), \mathrm{MWCNT}-\mathrm{COOH}(1 \mathrm{mg} / 100 \mathrm{~mL})$ and MWCNTTAP $(0.5 \mathrm{mg} / 100 \mathrm{~mL})$ in DMF solvent. Path length: $1 \mathrm{~cm}$.

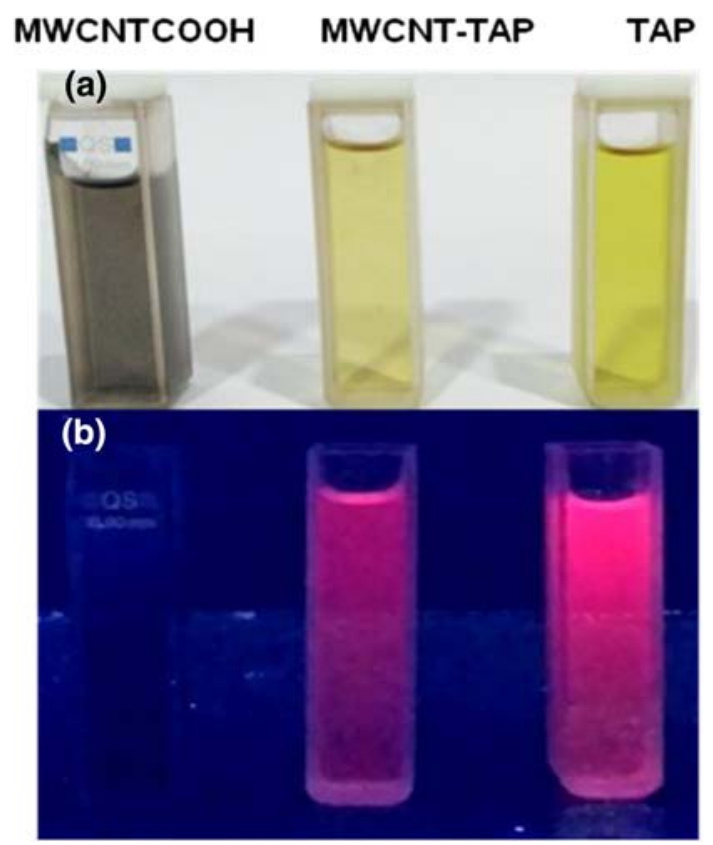

Figure 6. Images of MWCNT-COOH, MWCNT-TAP and TAP under (a) Visible light and (b) Long UV (365 nm).

not observed Van Hove singularities (VHS) as in the case of SWCNTs. This finding is similar to the literature report. ${ }^{38}$ MWCNT shows a peak at $265 \mathrm{~nm}$ in DMF solvent due to $\pi-\pi *$ transition. ${ }^{38}$ The absorbance at 268 $\mathrm{nm}$ and $429 \mathrm{~nm}$ of MWCNT-TAP was plotted against concentration to get a standard curve (see Figure S1 in Supporting Information, inset A and B, respectively, in $\mathrm{mg} \mathrm{L}^{-1}$ ). Similarly, we plotted the absorbance at 265 $\mathrm{nm}$ of MWCNT against concentration to get a standard curve as shown in Figure S2 (Supporting Information). We estimated the effective extinction coefficient of MWCNT and TAP in MWCNT-TAP from the slope of the linear least-squares fit to be $0.025 \mathrm{~L} \mathrm{mg}^{-1} \mathrm{~cm}^{-1}$ and $0.1102 \mathrm{~L} \mathrm{mg}^{-1} \mathrm{~cm}^{-1}$, respectively, with an $\mathrm{R}^{2}$ value of 0.998. Similarly, we found that the extinction coefficient of MWCNT from the slope of the linear least-squares fit to be $0.060 \mathrm{~L} \mathrm{mg}^{-1} \mathrm{~cm}^{-1}$ with an $\mathrm{R}^{2}$ value of 0.999 . Absorption coefficient of MWCNT varies due to different excited states. ${ }^{27}$ This method shows the much enhanced solubility. We estimated the amount of TAP in the MWCNT-TAP nanohybrid from the absorbance value $(\mathrm{A}=0.72)$ and the extinction coefficient of TAP in DMF solvent. The amount of TAP in $0.5 \mathrm{mg} / 100 \mathrm{~mL}$ of MWCNT-TAP solution is $0.318 \mathrm{mg}$. That is approximately $64 \%$ of hybrid mass is due to TAP. Therefore, the ratio of TAP to MWCNT in the hybrid is $1.7: 1$ $(\mathrm{g} / \mathrm{g})$.

The Soret band peak intensity of the MWCNT-TAP hybrid was suppressed compared to that of pure TAP. This result indicates that there is a charge transfer between TAP and MWCNT in the nanohybrid. This observation is well in accord with the Raman spectra. In order to confirm this, we have done detailed absorption studies in different solvents such as DMSO, DMF, methanol, DCM and THF. MWCNT-TAP nanohybrid shows good solubility in polar solvents such as DMSO, DMF, methanol and acetonitrile. Whereas, it is slightly less soluble in nonpolar solvents such as DCM, THF and ethyl acetate. However, after sonication it disperses well. We observed a bathochromic (or red) shift for both TAP and MWCNT-TAP in the absorption band with increasing solvent polarity as shown in Figure S3 (Supplementary Information). This type of bathochromic or red shift with increasing solvent polarity is usually termed as 'positive solvatochromism'. ${ }^{39}$ This shift is caused by differential solvation of the ground and first excited state of the chromophore. Positive solvatochromism will result if the excited state of the molecule is better stabilized by increasing the solvent polarity compared to the ground state. The Soret band absorption shifts for TAP and MWCNTTAP with increasing solvent polarity are tabulated in Table 1. Though methanol is more polar than THF and DCM, it shows blue or hypsochromic shift in owing to the ability of forming hydrogen bond with the methanol.

The covalently linked porphyrins on carbon nanomaterials can be employed as energy transporting antennae. Photoluminescent (PL) spectra of TAP and MWCNT-TAP nanohybrid in DMF solvent are shown in Figure 7. The solution of MWCNT-TAP nanohybrid exhibits $\sim 65 \%$ fluorescent quenching of emission 
Table 1. Soret band peak position of TAP and MWCNT-TAP in different solvents.

\begin{tabular}{lcc}
\hline Solvent with decreasing polarity & TAP (in nm) & MWCNT-TAP (in nm) \\
\hline DMSO & 438 & 430 \\
DMF & 436 & 429 \\
Methanol & 424 & 421 \\
THF & 430 & 426 \\
DCM & 428 & 425 \\
\hline
\end{tabular}

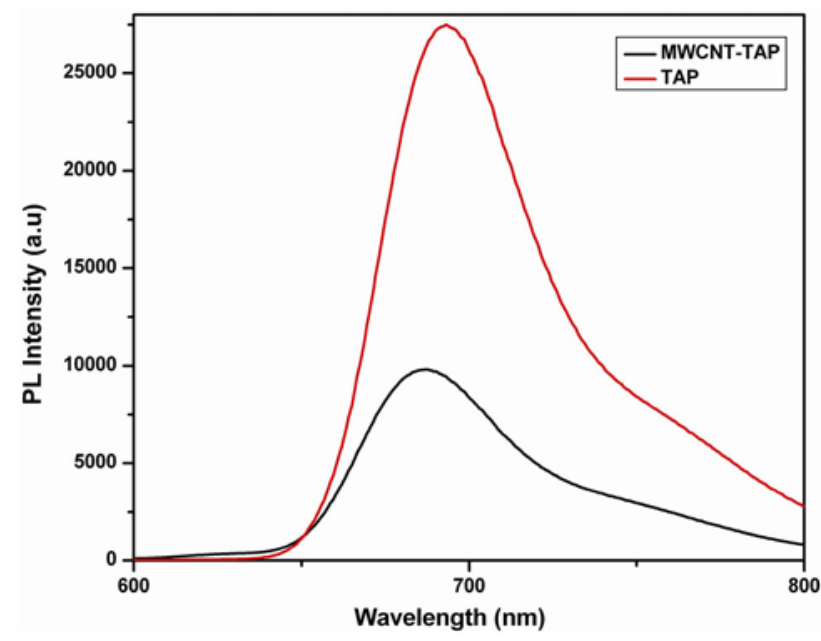

Figure 7. PL spectra of MWCNT-TAP $(0.17 \mathrm{mg} / 100 \mathrm{~mL})$ and TAP $\left(1.6 \times 10^{0-6} \mathrm{M}\right)$ in DMF $\left(\lambda_{e x}=450 \mathrm{~nm}\right)$ with the same absorbance value at the Soret band $(\mathrm{Abs}=0.24)$.

band at $693 \mathrm{~nm}$ as compared to that of pure TAP, upon excitation at $450 \mathrm{~nm}$. This peak is red shifted as compared to the reported porphyrin-functionalized carbon nanomaterials. ${ }^{21,22}$ This red shift is owing to the presence of electron donating amino group in the TAP moiety of MWCNT-TAP nanohybrid. Further, the substantial quenching confirms the covalent linking of porphyrins to carbon nanomaterials. This in turn substantiates the electron transfer from the singlet excited porphyrin to MWCNT. ${ }^{22}$ Upon excitation at different wavelengths (400, 430, and $550 \mathrm{~nm})$, MWCNT-TAP exhibits similar quenching as shown in Figures S4S6 (Supplementary Information) which also supports the electron transfer between TAP and MWCNT in nanohybrid. In order to confirm the electron-transfer between TAP and MWCNT in nanohybrid, we carried out detailed photoemission studies in different solvents such as DMSO, DMF, methanol and DCM at different excitation wavelengths (430 and $450 \mathrm{~nm}$ ). Similar quenching was observed for MWCNT-TAP in all these solvents as in DMF. DMSO exhibits $46 \%$ quenching whereas methanol and DCM exhibit $35 \%$ and $25 \%$,

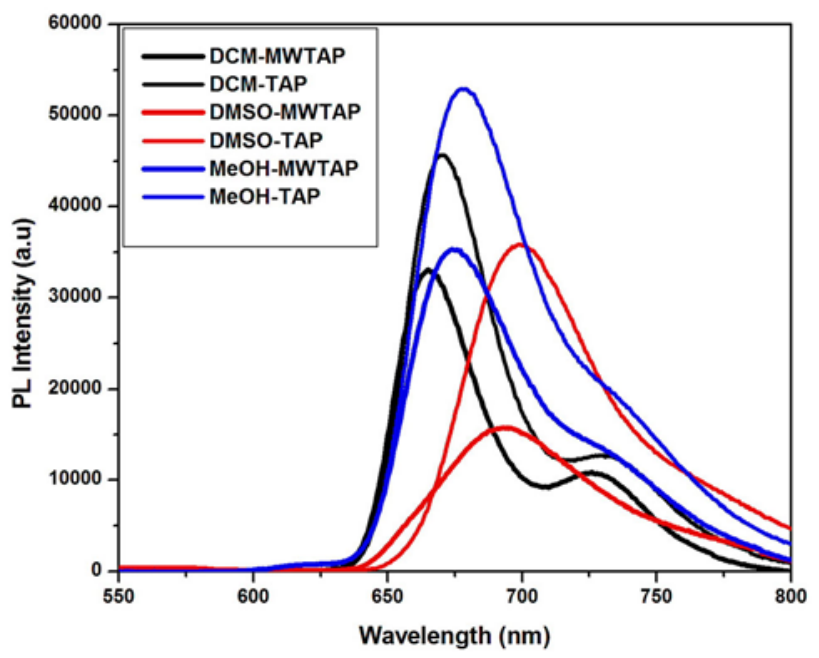

Figure 8. PL spectra of MWCNT-TAP $(0.17 \mathrm{mg} / 100 \mathrm{~mL})$ and TAP $\left(1.6 \times 10^{-6} \mathrm{M}\right)$ in different solvents $\left(\lambda_{e x}=430 \mathrm{~nm}\right)$ with the same absorbance value at the Soret band $(\mathrm{Abs}=0.24)$.

respectively, as shown in the Figure 8. Further, we observed bathochromic shift with increasing the solvent polarity, except in methanol which shows a blue shift. Generally, this kind of red shift is also termed as 'strong positive solvatochromism' which occurs in the dye molecules due to a large change in their permanent dipole moment upon excitation. A positive solvatochromism occurs when the solute dipole moment is higher in the excited state than in the ground state. The compounds with this particular type of behavior exhibit inter- or intramolecular charge transfer absorption. ${ }^{39}$ Apart from this dipole moment, the ability of a molecule to make hydrogen bonds with solvent molecules in its ground and excited states determines the extent and sign of solvatochromism. This is the reason for the blue shift in the emission and absorption spectra for both TAP and MWCNT-TAP in methanol. Blue shift in methanol is more pronounced in the absorption spectra compared to emission spectra. Quantum yield $\left(\Phi_{\mathrm{F}}\right)$ for the TAP and MWCNT-TAP were measured using TPP as reference at $20^{\circ} \mathrm{C}$; the measurement details are given in the Supporting Information. We found that the 
$\Phi_{\mathrm{F}}$ for TAP is 0.19 whereas the $\Phi_{\mathrm{F}}$ of MWCNT-TAP nanohybrid is 0.15 . The decrease in the $\Phi_{\mathrm{F}}$ is owing to the charge transfer between TAP and MWCNT in the hybrid.

\subsection{Morphology of MWCNTs and MWCNT-TAP}

The surface morphology of MWCNT and MWCNTTAP were studied using HR-TEM images as shown in Figures S7 and S8 (Supplementary Information). These images show the typical surface morphology of MWCNT before and after TAP functionalization. The relatively clear and smooth images of MWCNT surface became bumpy after TAP functionalization as shown in the Figure S8 (b) (Supplementary Information).

\section{Conclusions}

5,10,15,20-mesotetra(4-aminophenyl) porphyrin (TAP) was covalently functionalized with MWCNT through an amide linkage. The chemical structure of MWCNTTAP hybrid was confirmed by FT-IR spectroscopy. TGA curve of MWCNT-TAP reveals that there is a $\sim 60 \%$ weight loss in the range of $150-750^{\circ} \mathrm{C}$, which is attributed to the amount of TAP molecules that were attached to MWCNTs. The Raman spectroscopy indicates that the intensity of D band in MWCNT-TAP was significantly reduced compared with raw MWCNTs which reveal the improved electronic properties of MWCNTs in the nanohybrid. peak position of $\mathrm{G}$ band of the MWCNT-TAP nanohybrid exhibits a lower frequency shift compared with MWCNT. This lower frequency shift in the MWCNT-TAP hybrid confirms the charge transfer between TAP and MWCNT, which is very significant for high photocatalytic activity. Furthermore, emission spectra of nanohybrid show that there is fluorescence quenching of TAP by MWCNT in various solvents. This fluorescence quenching is due to the covalent functionalization of TAP with MWCNT molecules in the nano hybrid that facilitates the effective energy or electron transfer between porphyrin and MWCNT. The absorption and emission spectra of TAP and nanohybrid indicate strong positive solvatochromism with increasing solvent polarity. We found that the $\Phi_{\mathrm{F}}$ of TAP is 0.19 whereas the $\Phi_{\mathrm{F}}$ of MWCNTTAP nanohybrid is 0.15 . The decrease in the $\Phi_{\mathrm{F}}$ is owing to the charge transfer between TAP and MWCNT in the hybrid.

\section{Supplementary Information (SI)}

Calibration plot, TEM images of MWCNT and MWCNTTAP, UV-Vis spectra of TAP in different concentrations, PL spectra for different excitation wavelengths, (Figures S1-S8) and quantum yield measurement procedure are available at www.ias.ac.in/chemsci.

\section{Acknowledgements}

We gratefully acknowledge the financial support from COEAMGT (MHRD, New Delhi) for this research.

\section{References}

1. Ajayan P M 1999 Nanotubes from Carbon Chem. Rev. 991787

2. De Volder M F L, Tawfisk S H, Baughman R H and Hart A J 2013 Carbon Nanotubes: Present and Future Commercial Applications Science 339535

3. Baughman R H, Zakhidov A A and de Heer W A 2002 Carbon Nanotubes- the Route Toward Applications Science 297787

4. Wang F, Gu H and Swager T M 2008 Carbon Nanotube/Polythiophene Chemiresistive Sensors for Chemical Warfare Agents J. Am. Chem. Soc. 1305392

5. Kong J, Franklin N R, Zhou C, Chapline M G, Peng S, Cho K and Dai H 2000 Nanotube Molecular Wires as Chemical Sensors Science 287622

6. Guldi D M, Rahman G M A, Jux N, Balbinot D, Tagmatarchis N and Prato M 2005 Multiwalled carbon nanotubes in donor-acceptor nanohybrids-towards long-lived electron transfer products Chem. Commun.15 2038

7. Ehli C, Guldi D M, Herranz M A, Martín N, Campidelli S and Prato M 2008 Pyrene-tetrathiafulvalene supramolecular assembly with different types of carbon nanotubes J. Mater. Chem. 181498

8. Lu W, Li N, Chen W and Yao Y 2009 The role of multiwalled carbon nanotubes in enhancing the catalytic activity of cobalt tetraaminophthalocyanine for oxidation of conjugated dyes Carbon 473337

9. Rayati S and Sheybanifard Z 2016 Catalytic activity of $\mathrm{Mn}(\mathrm{III})$ and $\mathrm{Fe}(\mathrm{III})$ porphyrins supported onto multi-walled carbon nanotubes in the green oxidation of organic dyes with hydrogen peroxide: A comparative study J. Iran Chem. Soc. 13541

10. Wang X, Wang B, Zhong J, Zhao F, Han N, Huang W, Zeng M, Fan J and Li Y 2016 Iron polyphthalocyanine sheathed multiwalled carbon nanotubes: A high-performance electrocatalyst for oxygen reduction reaction Nano Res. 91497

11. Jin J, Dong Z, He J and Li R 2009 Synthesis of Novel Porphyrin and its Complexes Covalently Linked to Multi-Walled Carbon Nanotubes and Study of their Spectroscopy J. Ma. Nanoscale Res. Lett. 4578

12. Reddy A L M, Rajalakshmi N and Ramaprabhu S 2008 Cobalt-polypyrrole-multiwalled carbon nanotube catalysts for hydrogen and alcohol fuel cells Carbon $\mathbf{4 6} 2$

13. Wang A J, Fang Y, Long L L, Song Y L, Yu W, Zhao W, Cifuentes M P, Humphery M G and Zhang C 2013 Facile synthesis and enhanced nonlinear optical properties of porphyrin-functionalized multi-walled carbon nanotubes Chem. Eur. J. 153882 
14. Stylianakis M M, Konios D, Kakavelakis G, Charalambidis G, Stratakis E, Coutsolelos A G, Kymakis E and Anastasiadis S H 2015 Efficient ternary organic photovoltaics incorporating grapheme -based porphyrin molecule as a universal electron cascade material Nanoscale 717827

15. Rahman G M A, Guldi D M, Campidelli S and Prato M 2006 Electronically interacting single wall carbon nanotube-porphyrin nanohybrids J. Mater. Chem. 16 62

16. Li H, Zhou B, Lin Y, Gu L R, Wang W, Fernando K A S, Kumar S, Allard L F and Sun Y P 2004 Selective Interactions of Porphyrins with Semiconducting SingleWalled Carbon Nanotubes J. Am. Chem. Soc. 1261014

17. Murakami H, Nomura T and Nakashima N 2003 Noncovalent porphyrin-functionalized single-walled carbon nanotubes in solution and the formation of porphyrinnanotube nanocomposites Chem. Phys. Lett. 378481

18. Chen J Y and Collier C P 2005 Noncovalent Functionalization of Single-Walled Carbon Nanotubes with Water-Soluble Porphyrins J. Phys. Chem. B 1097605

19. Hasobe T, Fukuzumi S and Kamat P V 2005 Ordered Assembly of Protonated Porphyrin Driven by SingleWall Carbon Nanotubes. J- and H-Aggregates to Nanorods J. Am. Chem. Soc . 12711884

20. Yamuna R, Ramakrishnan S, Dhara K, Devi R, Kothurkar N K, Kirubha E and Palanisamy P K 2013 Synthesis, characterization, and nonlinear optical properties of graphene oxide functionalized with tetra-amino porphyrin J. Nanopart. Res. 151

21. Jin J, Dong Z, He J and Li R 2009 Synthesis of Novel Porphyrin and its Complexes Covalently Linked to Multi-Walled Carbon Nanotubes and Study of their Spectroscopy Nanoscale Res. Lett. 4578

22. Baskaran D, Mays J W, Zhang X P and Bratcher M S 2005 Carbon Nanotubes with Covalently Linked Porphyrin Antennae: Photoinduced Electron Transfer J. Am. Chem. Soc. 1276916

23. Moghadam M, Baltork I M, Tangestaninejad S, Mirkhani V, Kargar H and Isfahani N Z 2009 Manganese(III) porphyrin supported on multi-wall carbon nanotubes: A highly efficient and reusable biomimetic catalyst for epoxidation of alkenes with sodium periodate Polyhedron 283816

24. Xu Y, Liu Z, Zhang X, Wang Y, Tian J, Huang Y, Ma Y, Zhang X and Chen Y 2009 A Graphene Hybrid Material Covalently Functionalized with Porphyrin: Synthesis and Optical Limiting Property Adv. Mater. 211275

25. Li H, Martin R B, Harruff B A, Carino R A, Allard L F and Sun Y P 2004 Single-Walled Carbon Nanotubes Tethered with Porphyrins: Synthesis and Photophysical Properties Adv. Mater. 16896

26. Krishna M B M, Kumar V P, Venkatramaiah N, Venkatesan R and Rao D N 2011 Nonlinear optical properties of covalently linked graphene-metal porphyrin composite materials Appl. Phys. Lett. 98 081106-1

27. Guo Z, Du F, Ren D, Chen Y, Zheng J, Liu Z and Tian J 2006 Covalently porphyrin-functionalized single-walled carbon nanotubes: a novel photoactive and optical limiting donor-acceptor nanohybrid J. Mater. Chem. 163021

28. Liu Z B, Tian J G, Guo Z, Ren D M, Du F, Zheng J Y and Chen Y S 2008 Enhanced Optical Limiting Effects in Porphyrin-Covalently Functionalized Single-Walled Carbon Nanotubes Adv. Mater. 20511

29. Goyanes S, Rubiolo G R, Salazar A, Jimeno A, Corcuera M A and Mondragon I 2007 Carboxylation treatment of multiwalled carbon nanotubes monitored by infrared and ultraviolet spectroscopies and scanning probe microscopy Diamond Relat. Mater. 16412

30. Silva S, Pereira P M R, Silva P, Paz F A A, Faustino M A F, Cavaleiro J A S and Tome J P C 2012 Porphyrin and phthalocyanine glycodendritic conjugates: Synthesis, photophysical and photochemical properties Chem. Commun. 483608

31. Adler A D, Longo F R, Finarelli J D, Goldmacher J, Assour J and Korsakoff L 1967 A Simplified Synthesis for meso-Tetraphenylporphin J. Org. Chem. 32476

32. Qu K, Xu H, Zhao C, Ren J and Qu X 2011 Amine-linker length dependent electron transfer between porphyrins and covalent amino-modified single-walled carbon nanotubes RSC Adv. 1632

33. Tan J M, Karthivashan G, Arulselvan P, Fakurazi S and Hussein M Z 2014 Characterization and in vitro studies of the anticancer effect of oxidized carbon nanotubes functionalized with betulinic acid Drug Des. Devel. Ther. 82333

34. Datsyuk V, Kalyva M, Papagelis K, Partenios J, Tasis D and Siokou A 2008 Chemical oxidation of multiwalled carbon nanotubes Carbon 46833

35. Karousis N, Sandanayaka A S D, Hasobe T, Economopoulos S P, Sarantopouloua E and Tagmatarchis N 2011 Graphene oxide with covalently linked porphyrin antennae: Synthesis, characterization and photophysical properties J. Mater. Chem. 21109

36. Zdrojek M, Gebicki W, Jastrzebski C, Melin T and Huczko A 2004 Studies of multiwall carbon nanotubes using Raman spectroscopy and atomic force microscopy Solid State Phenomena 991

37. Chen Y, Huang Z H, Yue M and Kang F 2014 Integrating porphyrin nanoparticles into a 2D graphene matrix for free-standing nanohybrid films with enhanced visiblelight photocatalytic activity Nanoscale 6978

38. Han T K, Fen L B, Nee N M, Ahmad R and Johan M R 2011 Optical studies on multiwalled carbon nanotubes via modified Wolff-Kishner reduction process Adv. Mater. Res. 194-196 618

39. Reichardt C 1994 Solvatochromic Dyes as Solvent Polarity Indicators Chem. Rev. 942319 